\title{
L'impossible retour de Kossi Efoui : quand les racines africaines deviennent fantômes
}

\section{The Impossible Journey Home of Kossi Efoui's Solo d'un revenant, or the Destruction of the African Roots}

MAude LAfleur [lafleur.trudeau.maude@hotmail.com]

Université du Québec à Montréal, Canada

\section{RÉSUMÉ}

À l'approche du XXI ${ }^{e}$ siècle, la littérature africaine francophone se développe autour d'un schème principal : celui de la migration. Sachant que la plupart des auteur.e.s publié.e.s durant cette période vivent à l'extérieur du continent, principalement en France, la place que prend l'expérience de l'émigration n'est pas surprenante. On appelle ce nouveau courant littéraire la migritude. Même si le togolais Kossi Efoui est souvent associé à ce courant littéraire, les migrations qu’il met en scène semblent bien plus problématiques qu'une émigration transcontinentale typique et, par conséquent, elles soulèvent les importantes questions du rapport au passé et au futur, à la mémoire, de même qu'à la vie et à la mort. Dans cet article, nous étudierons le retour tel qu'il est représenté dans Solo d’un revenant (2008) de Kossi Efoui afin de dévoiler quelle vision de l'Afrique se révèle au fil des pages.

\section{MotS-CLÉS}

retour ; identité ; territoire ; littérature africaine ; migitude ; Kossi Efoui

\begin{abstract}
At the turn of the $21^{\text {st }}$ century, African literature of French expression evolves around a principal theme, that of migration. Knowing that a majority of these writers do not choose to live in their native country, but rather move to Europe, France more specifically, that propensity to write about their experience of migration is not surprising. The new literary movement that emerges during those years is thus called migritude. Even though Togolese author Kossi Efoui is often associated with that movement, the kind of migration he chooses to depict seems to be more complex than a typical transcontinental emigration and, therefore, raises important matters such as the relationships one develops to the past and the present, memory and history, and even life and death. This paper will explore the return home as it is represented in Kossi Efoui's Solo d'un revenant (2008) and will try to shed a light on the vision of Africa that the novels vehicles.
\end{abstract}

\section{KEYWORDS}

return; identity; territory; African literature; migritude; Kossi Efoui

REÇU 2015-08-31 ; ACCEPTE 2016-06-30 
À l'approche du XXI siècle, la littérature africaine francophone se développe autour d'un schème principal : celui de la migration. Sachant que la plupart des auteur.e.s publié.e.s durant cette période vivent à l'extérieur du continent, principalement en France, la place qu’y prend l'expérience de l'émigration n'est pas surprenante. On assiste alors à la production d'un discours culturel et littéraire qu’on baptise, en écho à la négritude, la migritude. Bien que les deux courants soient considérés comme en opposition, tous deux représentent un moment d'effervescence de la littérature francophone africaine et surtout une période de reconnaissance hors de l'Afrique pour les nombreux auteur.e.s qui les nourrissent. Les romans qu’on associe à la migritude traitent d'exil et de migration, souvent dans une perspective autofictionelle. S'inscrivant dans le courant de la littérature-monde (Le Monde, 16 mai 2007)' , ces romans mettent de l'avant un discours littéraire " décentré » (Chevrier 2008) et sans véritable ancrage géographique : "Les écrivains de la migritude tendent en effet, aujourd'hui, à devenir des nomades évoluant entre plusieurs pays, plusieurs langues et plusieurs cultures, et c'est sans complexes qu'ils s'installent dans l'hybride [...] » (Chevrier 2004 : 102). Ainsi, la quasi-totalité de ces textes n’ont pas pour lieu d'énonciation l'Afrique, mais plutôt l'Europe ou, dans quelques cas, l'Amérique. Le thème de la migration y est donc principalement exploité autour de la représentation d'une émigration transcontinentale et transculturelle. Ainsi, ces auteurs semblent caractérisés non par un déracinement tragique, mais par l'affirmation d'une double identité et une aspiration à une forme d'universalité.

Si l'auteur togolais Kossi Efoui est normalement associé au courant de la migritude², il s'éloigne des caractéristiques typiques du mouvement avec la parution de son roman Solo d'un revenant (2008). Bien que le roman mette en scène une migration, l'action se déroule entièrement au sein du continent africain. Le narrateur du roman retourne chez lui lors de l'ouverture des frontières entre le Nord et le Sud d'une ville africaine imaginaire nommée Gloria Grande. Originaire du Sud, il se trouvait au nord de la frontière alors qu'un conflit armé a éclaté et l'a empêché de rentrer chez-lui. Après dix ans de massacres, il attend l'autorisation de rentrer chez lui près de la frontière engorgée dans le but très précis de confirmer ses suspicions quant à l'implication de son ami dans les massacres et de le punir si elles s'avèrent vraies. Bien que le

1 L'année 2006 fait la preuve du bouleversement qu’est en train de vivre le paysage littéraire francophone alors que grand nombre de prestigieux prix littéraires français sont remis à des auteurs qui se sont pas originaires de l'Hexagone. (Cette année-là, le Congolais Alain Mabanckou remporte le Prix Renaudot pour son roman Mémoires de porc-épic; l'Américan Jonathan Littell le Goncourt et Grand Prix du Roman de l'Académie française pour Les bienveillantes; la Camerounaise Léonora Miano le Goncourt des lycéens pour Contour du jour qui vient et la Canadienne Nancy Huston se voit remettre le prix Femina pour Lignes de failles.) Cette ouverture du centre littéraire français vers les francophones d'ailleurs se confirme dans les années suivantes alors que les auteur.e.s des périphéries continuent d’être récompensé.s. C’est dans cette foulée que parait le manifeste pour une littérature-monde, qui porte la signature de nombreux auteurs renommés tel que le lauréat du prix Nobel de $2007 \mathrm{~J}$. M. Le Clézio. Ce texte engagé proclame venue lère, une ère où lécrivain libéré du nationalisme géographique et prône « la reconnaissance et la prise de conscience de notre apport à l'intelligence humaine, avec cet outil qu'est la langue française " (Lebris et Rouaud 2007 : 65). Ainsi, les signataires réclament le décentrement de l'institution et l'élimination de la hiérarchie entre la production culturelle française et francophone.

2 Comme Solo d'un revenant, La fabrique des Cérémonies (2001) raconte aussi l'histoire d'un migrant africain : Edgar Fall, le personnage principal, est un Togolais qui étudie URSS et qui, suite à la chute du mur de Berlin, se déplace plutôt vers la France. Là, il sera contacté par un ancien camarade de classe qui lui proposera de participer à la rédaction d'un magazine de voyage. À cette fin, il retourne alors dans son pays d'origine, mais le retour de Fall est, encore une fois, problématique. 
contexte du roman soit imaginaire plutôt que référentiel, plusieurs critiques y ont reconnu les dynamiques du génocide rwandais. En mettant en scène une telle migration, Efoui séloigne d'un lieu commun de la littérature africaine de la migritude et représente alors une quête qui diffère en plusieurs points de la quête identitaire du sujet postcolonial tel qu'il peut être représenté chez Le Clézio, Fatou Diome, Henri Lopez ou Léonora Miano, pour ne nommer qu'eux. Cet article explorera donc la tension portée par le roman d'Efoui, c'est à dire la confrontation de deux identités culturelles de l'inconfort développées en parallèles : celle, collective et en transformation, d'une population au sortir d'une crise majeure et celle, en constant décalage, d'un individu exilé. Malgré l'écart entre la migration typique et celle dépeinte par Efoui, la position occupée par le narrateur-revenant n'est nulle autre que celle de l'écrivain de la migritude devant une Afrique qu'il veut quitter.

Dès le départ, le roman d'Efoui confronte deux communautés, celle de Sud Gloria, d'où le narrateur est issu, et celle de Nord Gloria, demeurée en paix. C'est au sein de cette dernière que le personnage s'est réfugié, exilé malgré lui. Le narrateur devient ainsi le lieu de contact, le seuil entre ces deux communautés. Il devient le lieu de l'identité ou, comme l'écrit Homi Bhabha (cité par Moura 1999) «un site de la construction du sens - qui déplace non seulement les termes de la négociation mais permet d'inaugurer une interaction ou un dialogisme dominé/ dominant» (166) qui permet la création d'une identité autre, hybride. Lémigration européenne telle qu'elle est souvent présentée dans les romans de la migritude, force le sujet à la construction d'une nouvelle catégorie identitaire qui lie les deux contextes culturels duquel il est issu. Citons à cet effet l'exemple de Léonora Miano, romancière et essayiste d'origine camerounaise ayant beaucoup réfléchi sur a question de l'appartenance; ses écrits illustrent bien ce besoin de lier la quête identitaire du personnage africain autant à l'Europe qu'à l'Afrique puisque les deux milieux jouent un rôle clé dans la constitution d'une appartenance culturelle et personnelle. Elle publie, en effet, deux trilogies qui témoignent de l'importance que prennent le pays d'origine et le pays d'accueil : la première qu'elle nomme Suite africaine et la seconde qui a pour décor la ville de Paris. Dans ses essais, Miano précise sa position particulière en se disant ni exclusivement européenne, ni simplement africaine, mais « afropéenne » : " ma propre identité, je la dis frontalière, ancrée non pas dans un lieu de rupture, mais, au contraire, dans un espace d'accolement permanent » (Miano 2012 : 25). C'est ainsi, que l'écriture devient, pour Miano, un lieu d'articulation et de contact qui permet la production d'une identité hybride. Ainsi, l'inconfortable entre-deux (Bhabha 2007) devient un lieu positif, permettant la transformation et le double enracinement du sujet.

Cependant, Kossi Efoui dans son roman Solo d'un revenant nous présente un personnage exilé qui retourne dans son pays d’origine après une décennie. Dans ce cas, la terre d'exil et le lieu d'origine sont deux zones d'une même ville africaine qui se positionnent, non pas dans un rapport de contiguïté comme la France et le Cameroun pour Miano, mais se développe en tant qu'antagonistes. Nord et Sud Gloria résultent de la division d'une même communauté et se sont opposés dans un conflit politique d'envergure. Du fait, on ne peut appartenir à la fois à Nord et à Sud Gloria comme Léonora Miano devient « afropéenne ». Le retour du narrateur dans sa ville natale et la quête qu'il entreprend représente donc une articulation identitaire qui diffère de celle du migrant francoafricain typique. Le revenant d'Efoui doit, en effet, mettre en relation une identité individuelle en déconstruction et une identité collective basée sur des souvenirs 
qui ne trouvent plus leurs échos dans la société actuelle. Les deux identités qui se jouent en lui et qui nourrissent la tension du motif du retour ne sont pas réductibles à une seule identité cohérente. Mais, encore plus que des questions identitaires, le motif du retour qui sous-tend le roman d'Efoui pose la question du visible et de l'invisible. Dans le cadre de cette étude, nous nous attarderons à la signification de la migration et plus particulièrement du retour, dans le roman d'Efoui et dans sa relation au courant littéraire de la migritude. Pour ce faire, nous étudierons d'abord les obstacles et frontières qui entravent le déplacement du narrateur et qui témoignent de la division du territoire comme d'une mesure de contrôle de l'individu.

La longue absence du narrateur hors de sa ville natale n'est pas volontaire ; il est maintenu en exil dans une communauté qui n’est pas la sienne par un ensemble de dispositifs de sécurité qui renforcent la non-perméabilité des frontières. Ainsi, et même lors de la réouverture de la frontière, les autorités se munissent d'un système juridico-légal qui limite les déplacements ainsi que de mécanismes disciplinaires qui concentrent et contraignent la population. Il est alors possible de voir les marques du pouvoir et de la discipline, tels que les a pensés Michel Foucault dans les descriptions du territoire mis en scène par Efoui dans son roman. « Le premier geste de la discipline ", affirme Foucault (1993), "c'est [...] de circoncire un espace dans lequel son pouvoir et les mécanismes de son pouvoir joueront à plein et sans limites » (46). Autrement dit, pour que le processus de contrôle fonctionne et que l'individu subisse le sort auquel on le destine, l'espace est alors planifié et divisé :

À chaque individu, sa place, et en chaque emplacement, un individu. [...] L'espace disciplinaire tend à se diviser en autant de parcelles qu'il y a de corps ou d'éléments à répartir. Il faut annuler les effets de répartitions indécises [...] La discipline organise un espace analytique. (Foucault 1993 : 168)

Cette division de l'espace repose sur une ensemble de procédures qui répondent à un code officiel et servent un effet bien précis, ce que le roman a tôt fait de démontrer :

L'agent en uniforme local suspendu au laissez-passer, un carnet orange et bleu pétrole, ouvert à la page où le cachet de reconnaissance faisant foi d'un rouge officiel s'accroche convenablement au gros plan de la tête sur la photo. Il faut imaginer qu'il veille sur la bonne mesure de l'angle que compose le poignet avec l'avant-bras lorsque le document est correctement tenu et tendu de façon règlementaire. (Efoui $2008: 18-19$ )

Chaque geste de l'agent est calculé et chaque décision régie par le système disciplinaire qu'il sert sans réfléchir, prouvant ainsi qu'il est utilisé à titre d’opérateur ${ }^{3}$ de la discipline. L'ensemble de procédures qui divise le territoire en zones exclusives, contrôle le déplacement au moyen d'un visa marque le disciplinaire en imposant un interdit bien plus qu'offrant une permission. Ainsi la carte qui permet aux exilés de revenir à domicile et qui est présentée comme un privilège se rapproche,

3 Selon Gil (1985), l’opérateur est « un dispositif technique qui transforme la force et la conserve [...] » (19). À la fois expressif, communicatif et actif, il pré-signifie la force, la communique sans la modifier et, finalement, produit un effet sur une autre force. Les opérateurs sont des outils techniques parmi lesquels on compte les armes et autres objets, mais également le corps lui-même. 
dans les faits, bien plus d'une technique de contrôle et de surveillance. En effet, pour les quelques personnes dont le retour est autorisé, il y en a bien plus à qui on répète "Débrouille » (13) ou encore à qui on commande par les haut-parleurs de « laisse[r] passer quelques jours » (13).

L’ensemble juridique qui accompagne la ségrégation s’établit comme l'application d'une force qui marque d'emblée l'ordre spatial. Cette division du territoire, qui parcourt le roman d'Efoui, pose déjà un premier obstacle, physique, au retour du narrateur. Cet espace divisé sur un mode disciplinaire marque une limite à ne pas franchir et restreint le mouvement des individus. Lexil vécu par ceux qu'on appelle « les déplacés de longue date » (27) est prolongé par la lourdeur des procédures et modifie le sentiment d'appartenance qui se construit par rapport à un chez-soi lointain, isolé et avec lequel ils ont perdu contact.

C'est d'ailleurs la transformation du milieu, celle là-même qui empêche le sentiment d'appartenance du narrateur, qui permet l'ouverture de la frontière et rend possible son retour. Ce qu'on constate en entrant à Sud Gloria, c'est l'image d'un pays en reconstruction, en processus de pacification : "La bande d'anciens chasseurs de têtes et coupeurs d'organes imitant bravement le sourire du coach belge, imitant le sourire comme il faut pour demander les papier et les rendre, comme le veut la coutume dans les sociétés libres qu’on appelait autrefois civilisées » (24). Ainsi, tour à tour, toutes les figures de combattants sont pacifiées, elles sont déchargées de leur violence, rendues inoffensives et récupérées par l’appareil disciplinaire. Mais on voit bien, à l'ironie du ton qu'emploie Efoui, que la pacification sonne faux, comme si elle nétait performée que dans les apparences et comme si elle demandait à ce qu'on y adhère comme à une fiction. Cependant, louverture des frontières marque bien le passage officiel de la guerre à la paix et ce passage est essentiel à la migration du narrateur. Le retour des exilés marque l'imaginaire collectif et fait écho à la pacification du pays :

Un retour triomphalement salué par des photographes de presse au moment où l'on atteint sur le dernier point de passage, l'épais mur blanc sur lequel le mot checkpoint a été barré et remplacé par l'inscription BIENVENUE [...] où un polisson avait rajouté AUX REVENANTS. BIENVENUE AUX REVENANTS. (28)

Encore une fois, c'est dans les apparences, dans la matérialité de la ville, que la transformation qui permet l'accueil des revenants transparait. Par contre, cette inscription peinte à l'entrée de Sud Gloria ne signifie pas que les revenants seront réintégrés à la collectivité sans heurt. Dès le début, le roman laisse percevoir un hiatus entre l'identité individuelle et l'identité collective : " on finira par comprendre que l'odeur des forêts n'est plus celle des arbres » (12). Ainsi, Efoui souligne l'incompatibilité entre la collectivité et les individus qui la composent. La négation " n'est plus » marque le passage d'une ère à une autre et réaffirme la transformation de la collectivité. Dans cette nouvelle ère, l'identité qui est mise de l'avant par la collectivité, n'est plus soutenue par les individus qui la réintègrent, parce que si l'individu est mobile, la communauté, elle, ne l'est pas. La sédentarité, qui ne favorise pas la rencontre de l'autre, et les normes qui régulent le vivre ensemble d'une collectivité en fixent l'identité et toute transformation potentielle est grandement ralentie. Il en résulte donc un hiatus ou un débalancement ; les catégories qui forment la collectivité et en répartissent les membres ne correspondant plus aux individus qu'elles se doivent de représenter. 
L'identité collective mise de l'avant par la migritude apparaît difficile à saisir, ou du moins changeante. La fracture de l'identité personnelle du personnage principal semble être en fait un miroir de ce flou identitaire qui sévit dans la société référentielle, puisque, bien que le roman se déroule dans une ville imaginaire nommée Gloria Grande, le conflit rappelle et universalise les dynamiques du génocide rwandais. Toujours tiraillé entre deux influences ou deux appartenances, le narrateur anonyme se trouve dans une position d'inconfort, celle de l'entre-deux. Dans cette situation, affirme Homi Bhabha (2007) « les frontières deviennent le foyer, et le monde devient confus » (41). La position du migrant, pour autant qu'elle permet l'échange, est également inquiétante et tout un ensemble de conditions est nécessaire avant de tirer profit de l'hybridité qui caractérise le courant de la migritude. Contrairement aux romans mettant en scène une migration transatlantique, le parcours du personnage d'Efoui ne permettra pas de « reconnaitre un moi pluriel, formé par le dialogue permanent entre l'" individu ethnique (ethnic self) et la culture dominante " (Moura 1999 : 166). La violence qui caractérise "les dix années de massacres " (Efoui 2008) teinte, bien évidemment, le rapport du narrateur à sa terre natale. Nous examinerons comment l'inconfort de cette fracturation du soi devient une conséquence de la migration forcée et de l'exil et surtout, nous tenterons de démontrer que le retour ne représente pas, chez Kossi Efoui, une solution ou une libération. Le projet identitaire de l'hybridité est voué à l'échec et la position désincarnée du revenant devient la preuve de l'impossibilité d'articuler ces deux pôles irréconciliables. De ce fait, il assume une posture de passeur, une posture de l'entre-deux.

En nommant ses exilés « revenants », Efoui joue sur une double signification qui traduit bien le rôle que joue le narrateur tout au long du roman. Signifiant à la fois celui qui revient, mais convoquant également l'imaginaire du fantôme, le rôle que joue le revenant-narrateur au sein $\mathrm{du}$ récit se révèle justement double. Le revenant est une figure qui se tient sur le seuil entre la vie et la mort, entre le passé et le présent, entre le réel et l'iréel ${ }^{4}$ et, dans le cas du narrateur entre deux identités. En effet, n’appartenant ni à la communauté du Nord, ni à celle du Sud à cause de la longue période de l'exil à laquelle il a été soumis, le narrateur met en lumière le rejet de sa communauté lors de retour. Dès lors, le narrateur fait face à « la croyance publique voulant que toute personne installée dans le Nord avant la guerre ait forcément profité de l'enrichissement de la zone par le trafic d'armes, [...] est donc riche, et comme telle a une dette illimitée à l'égard de ceux de ce côté-ci et qui répètent fièrement : tu n’oublie pas, nous, nous sommes un pays en guerre " (58). L'expression "nous, nous sommes un pays en guerre " qui martèle le roman signale une tension entre deux communautés distinctes et impose des conditions à l'appartenance collective ${ }^{5}$ qui se construit autour d'une expérience exclusive, celle de la guerre.

Il y a donc un hiatus entre les mots visibles, ceux écrits à la craie sur le mur qui sépare Nord Gloria et Sud («BIENVENUE AUX REVENANTS »), et les mots invisibles qui expriment l'opinion d'une collectivité qui se construit autour d'une expérience commune dont les exilés sont

4 Le brouillage des frontières entre le réel et l'irréel et entre la vie et la mort s’effectue dès l'exergue alors qu'Efoui lance, au lecteur, cet avertissement : « Les personnages de ce livre sont des êtres de fiction comme nous tous. Toute ressemblance, même fortuite, avec les vivants, les morts et les morts vivants, est donc bien réelle ».

5 Nadine Gordimer (1988) avance que l'acceptation des pairs, de même qu’un désir d'appartenance, sont les deux conditions nécessaires à l'intégration d'un individu à un groupe : « But belonging to a society means : desire to belong and acceptance from others $»(32)$. 
exclus. Ce n'est pas un hasard si seul le personnage du narrateur, le revenant, arrive à révéler les incohérences qui fissurent le monde autour de lui. Condamné à évoluer sur le seuil et dans les interstices, le revenant développe une vision du monde dissidente, presque schizophrénique. Dans une monographie consacrée à la figure du revenant, Martine Delvaux (2005) affirme que : «Devant lui, il ne peut y avoir que de l'incertitude et de la foi. Jamais celui qui voit le spectre ne sera rassuré, car toujours ce regard sera impossible à croiser [...]» (16). Le revenant qui est mis en scène par Efoui n'échappe certainement pas à la règle ; effrayant et inquiétant, son regard révèle les incongruités qui échappent à ceux qui font partie de la communauté de Sud Gloria. L'entre-deux qu'il habite devient le seul lieu où se superposent des positions irréconciliables.

Dès qu'il franchit la frontière, le narrateur force la cohabitation du passé et du présent :

Il faut imaginer autrefois, au bout de cette rue, les échoppes de ceux qui ont profession de guérir, leur boutiques à médicaments où étaient étalés des flacons de couleur [...]. Et devant les échoppes, l'encombrement d’enseignes. [...] On voit à présent la rare échoppe d'une coiffeuse réduite à l'enseigne et au tabouret sur le trottoir [...]. (29-30)

Le revenant force ainsi la superposition du passé et du présent en un seul espace, celui de l'imaginaire. Du fait, le lieu convoqué relève du registre de l'inquiétante étrangeté à la fois familier et étranger au narrateur. La même impression persiste lorsqu'il se rend à la maison du seul ami qui lui reste dans le pays : Asafo Johnson. Dès qu'il aperçoit la demeure, il commente : "La maison a survécu » (30). Le choix du participe passé « survécu » dévoile la particularité du regard du narrateur et insuffle à la maison une existence propre, une vie. Comme la maison, le décor convoqué par l'imaginaire du narrateur semble être figé à mi-chemin entre deux époques ou deux états. Il poursuit : « [...] je reconnais la cour, je reconnais le manguier. Cette maison était autrefois grouillante de chiens. Et sans leur présence sonore, quelque chose me manque pour croire entièrement à la réalité » (32). L'extrait démontre bien le hiatus provoqué par l'expérience du revenant, la distance entre le passé et le présent déformant sa perception et l'empêchant d'appréhender complètement sa nouvelle réalité. Comme un fantôme, le revenant n'a pas de chez-soi. Le lieu qui devient le site de son apparition est une demeure qui n'en est pas une. Le fantôme est sans lieu propre, sans résidence (home) et, comme le démontre sa position, il hante une réalité sur laquelle il n’a pas de prise. Cette incapacité à reconnaître et donc à avoir prise sur le monde qui l'entoure lève le voile sur des failles, des fissures dans le tissu de la réalité6. Dans son rapport au monde, le narrateur-revenant dévoile une falsification, une altération invisible pour les autres : "Il se pourrait bien, se dit le revenant, si je me retournais soudain, que tout ce leurre s'efface comme crève l'enveloppe d'une image. Et ça ne me prendrait qu'une

6 Le narrateur de La fabrique des cérémonies, un autre romans d'Efoui, entretient un rapport similaire à son pays d’origine lors de son retour au Togo tel que le relève Sophie Lavigne (2011) : « La frontière la plus importante est la FRONTIĖRE ENTRE LE RÉEL ET L'IRRÉEL, qui a 30 références pour $74 \%$ du texte. Cette frontière décrit principalement le flou dans lequel notre héros baigne, tout est étrange, hors norme, comme un cauchemar. La FRONTIËRE ENTRE LA VIE ET LA MORT compte 18 références qui couvrent $39 \%$ du texte. Cette frontière est parfois croisée avec la frontière entre le réel et l'irréel. Elles sont toutes deux colorées par l'horreur lorsqu'elles sont regroupées. La FRONTIËRE ENTRE NOUS ET L'AUTRE compte 17 références qui couvrent $24 \%$ du texte ; cette frontière met en scène l'incompréhension de notre héros avec sa terre natale. La mort et la précarité sont les enjeux fondamentaux du propos de l'auteur et elles sont partout lors du voyage au Togo » (64). 
fraction de seconde pour atteindre le réveil et me retrouver à Nord Gloria que je n'aurais peutêtre jamais quitté » (33).

Cette idée d'un leurre ou d'une tromperie met en lumières les travers de la relation entre le passé et le présent et sous-entend que, bien que la guerre soit terminée, la pacification du territoire n'est atteinte. Ainsi permettre le retour des exilés entretient le leurre et alimente l'illusion d'une paix qui n'existe pas. C’est donc la question de la mémoire qui est ici mise en scène dans ce territoire pacifié où la violence demeure comme en sourdine dans l'histoire collective, vacillant entre désir d’oublier et devoir de mémoire. Du fait, le narrateur se retrouve dans un pays en immédiat après-guerre, mais où la violence est invisible, insaisissable. Quand elle refait surface, c'est dans un langage qui appartient à l'univers merveilleux et surnaturel du conte : "Présences armées » autour des points d'eaux dont parlaient les gens de la Longue Fuite./ Comme on parle d'une apparition./ On aurait cru à une vision sortie de ces contes terribles qu'on dit aux enfants sages » $(85)^{7}$. La violence devient une apparition, quasi-fantomatique elle aussi. À la manière des chiens qui peuplaient jadis la cour de son ami, c'est à travers les perceptions auditives qu'on découvre la violence, mais jamais par la vue, comme une rumeur dont la véracité n'est jamais confirmée.

Il a été dit de ce roman qu'il réactualisait les dynamiques du génocide rwandais ${ }^{8}$. Par contre, en séloignant du témoignage ou du récit qui ont été au centre de cette production littéraire, Efoui pose cette question de la mémoire bien différemment. Déjà, en ne nommant pas le pays qu'il met en scène, il universalise les dynamiques de domination et de violence et les intemporalise. De ce fait, Efoui s'éloigne d'une littérature témoignage qui agit à la manière d'un documentaire, il démontre le potentiel réflexif de l'imaginaire littéraire. Cependant, Efoui nie le rapprochement possible entre la violente histoire du Rwanda et l'intrigue de Solo d'un revenant. Comme le confirme Boco (2013), « Kossi Efoui affirme que son œuvre ne parle pas du Rwanda, mais de la défaite de l'homme, un sujet à caractère universel » (110). Ainsi, comme les auteur.e.s de la migritude, Efoui tend vers une universalité, vers un effacement des frontières géographiques, et ira même jusqu'à dire que, surtout dans le cas des romans de la migritude, la littérature africaine n'existe plus vraiment comme elle tire maintenant ses racines loin du pays natal : "La littérature africaine n'existe pas. [...] L'écrivain africain n'est pas salarié par le ministère du tourisme, il n'a pas mission d'exprimer l'âme authentique africaine " (Efoui cité par Boco 2013 : 20). Par contre, même s'il se réclame d'une littérature-monde, le roman d'Efoui peint tout de même une réalité propre à l'Afrique. Ainsi, le lieu dans lequel se déroule l'intrigue, bien qu'il soit une invention de l'auteur, est vital au récit. Nous avons déjà démontré l'importance accordée au rapport du narrateur au territoire. Ainsi, la position du revenant n'est nulle autre que celle de

7 La mise en page de ce passage diffère d'ailleurs de celle de la majorité du roman ; Efoui met alors à profit la versification. Ainsi, alors que le propos rappelle l'univers du conte, la présentation visuelle renvoie plutôt à celui de la poésie. Ce processus a pour effet de construire une autre barrière, cette fois-ci formelle, entre le lecteur et la violence représentée.

8 «Il est important de noter ici que l'histoire de violence que l'auteur togolais révèle à travers le passage ci-dessus mentionné où des citoyens d'une même nation sont considérés comme des étrangers et de ce fait deviennent la cible d'extermination, semble présenter tous les prototypes du génocide de 1994 au Rwanda, en Afrique de l'est; génocide au cours duquel plus d'un million de Tutsis étaient tués, la plupart à coups de machette par leurs propres compatriotes, les Hutus, en seulement 100 jours » (Bocco 2013 : 108). Dans le roman, il s'agit en effet de la même ville Gloria Grande qui est séparée en deux zones opposées : le Sud et le Nord. 
l'auteur de la migritude, forcé de faire sens d'un territoire étrange, étranger et fuyant. L'Afrique que nie Efoui est une Afrique en déconstruction dont nous parlent les écrivains de la migritude, nés après les indépendances. Ainsi, s'il n'existe pas de littérature africaine pour Efoui, c'est que l'Afrique existe seulement au passé. Il devient alors impossible d'y prendre racine et l'Afrique apparaît, sous la plume d'Efoui et de ses compatriotes, comme un No man's land: un lieu de non-sens et de non-avenir. Il devient alors nécessaire aux écrivains de la migritude de trouver un autre lieu pour donner corps à leurs personnages; il faut, soit le puiser ailleurs ou alors à même son imagination ${ }^{9}$. Par contre, que l'action soit située dans un pays africain imaginaire ou dans un pays européen réel, l'absence d'une Afrique référentielle en dit plus long sur le rapport de l'auteur au continent que sur le lieu représenté. La disparition devient un symbole d'un rapport nostalgique à un passé que les auteurs n'ont pas connu, la responsabilité d'une mémoire douloureuse et d'un retour (dans le temps) impossible. D'autant plus que le refus d'oublier la violence $^{10}$ et de se détacher du passé qui caractérise le roman d'Efoui participe du « devoir de mémoire " qui a été le mot d’ordre de la représentation littéraire du Génocide Rwandais. En ce sens le roman pose une question vitale, comment construire un avenir stable sans renier les blessures du passé ? Comment préserver la mémoire du passé sans le recréer ? Comment les africains peuvent-ils prendre racine dans un sol brûlé et fleurir simultanément ?

En plus d'être perpétuellement entre deux états, le revenant est un être sans repos, dans une quête perpétuellement inachevée. Le lecteur apprend en effet, au fil du roman, à reconstituer la quête du narrateur et comprend que celui-ci revient au pays dans le but de trouver Asafo Johnson, son ancien ami. De Nord Gloria, le narrateur avait eu accès à des bribes d'information, des « indices » (Efoui 2008), qui lui avaient appris que Johnson était impliqué dans le génocide qui a couté sa vie à un ami cher. Dès son retour, le narrateur entreprend donc de retrouver Johnson, de confirmer ses suspicions et de le tuer pour venger la mort de leur camarade. L'enquête du narrateur prend peu à peu la forme d'une réhabilitation et fait écho à l'état de Sud Gloria dont les citoyens accomplissent les «actes de la reconstruction » (26). Sa quête reprend les dynamiques qui motivent l'ambition collective et s’étend, par conséquent, plus loin qu'une simple quête personnelle. Sous la plume d'Efoui, il revient au revenant de rétablir l'ordre, d'assurer la reconstruction et la réconciliation en éliminant les éléments qui entravent la pacification : « J’ai levé l'arme. J'ai visé tous ces bruits errants, j’ai visé toutes ces voix, j'ai visé dans le tas, tous ces être sonores » (200). Dans cette phrase, la position du revenant devient claire, il appréhende l'invisible et le visible et les combine puisqu'en ayant dans la mire Asafo Johnson, un individu bien réel sur le plan narratif, il pointe en fait son arme sur l'invisible qui gangrène la société. Mais, étant le symbole de l'instabilité qui caractérise Sud Gloria et l'Afrique en déconstruction, le revenant ne trouve pas le repos et ne peut mener à bien sa quête. Après tout, comme l'indique la citation d'Henri Michaux mise en exergue de la seconde partie du roman, « [i]l serait

9 Nombreux sont les auteur.e.s qui choisiront, comme décor à leur intrigue, un pays africain imaginaire (Sony Labou Tansi, Léonora Miano, Amhadou Kourouma, etc.).

10 Ce devoir de mémoire est vu comme un espoir, cependant vain, de rompre le cycle de violence duquel le continent entier est prisonnier. L'univers représenté est donc sans futur et cette mise en scène contribue à alimenter l'impression d'un monde dans lequel on ne peut prendre racine, un monde « où les morts n'en finissent plus d'enterrer leurs morts, où les descendants de victimes s'en vont apprendre, de génération en génération, quelque chose sur les armes qui auront blessé leurs ancêtres, jusqu'à la génération qui apprendra à manier à son tour les même armes, dans le sens de la rétribution, avec le même art de désigner l'ennemi, avec la même nostalgie d'un pur commencement » (27). 
bien extraordinaire que des milliers d'événements qui surviennent chaque année résultât une harmonie parfaite Il y en a toujours qui ne passent pas, et qu'on garde en soi, blessants. Une des choses à faire : l'exorcisme ». C'est donc une Afrique dystopique qui se délite de page en page que nous présente Solo d'un revenant et le roman devient l'exorcisme d'un monde disharmonieux, dissonant et inhumain. Par l'écriture, Efoui refuse la reconstruction qui impose un impératif de l'oubli, de la table rase et, en faisant un détour par l'imaginaire, il impose à ses lecteurs un difficile devoir de mémoire. Ainsi, le destin du revenant est diamétralement opposé à celui du migrant européen tel qu'il est présenté dans la majeure partie des romans de la migritude, un personnage qui s'épanoui hors de l'Afrique et qui développe une double appartenance. C'est le récit d'un échec que nous fait Efoui, celui de l'impossible retour, celui d'un héros qui échoue sa quête de vengeance, celui d'un peuple qui prend racine dans une terre en décomposition, d'un peuple qui ne semble pouvoir pas refleurir de sitôt.

\section{Références bibliographiques}

Bhabha, H. K. (2007 [1994]). Les lieux de la culture : une théorie postcoloniale. Paris : Payot.

Bocco, A. (2013). Kossi Efoui ou la perspective d'un nouvel engagement: le pouvoir d'exorcisme de l'écriture dans Solo d'un revenant et L'ombre des choses à venir. PhD diss., University of Tennessee, <URL : http://trace.tennessee.edu/utk_graddiss/1697>

Chevrier, J. (2004 [1974]). La littérature nègre. Paris : Armand Colin.

Chevrier, J. (2008). La littérature africaine : une anthologie du monde noir. Paris : Librio.

Delvaux, M. (2005). Histoires de fantômes - spectralité et témoignage dans les récits de femmes contemporains. Montréal, Les Presses de l'Université de Montréal. ProQuest ebrary. Web. 12 March 2015

Efoui, K. (2008). Solo d'un revenant. Paris : Seuil.

Foucault, M. (1984). Des espaces autres (conférence au Cercle d'études architecturales, 14 mars 1967), Architecture, Mouvement, Continuité, 5. <http://desteceres.com/heterotopias.pdf>

Foucault, M. (1993). Surveiller et punir : naissance de la prison. Paris : Gallimard. . (2004). Sécurité, territoire, population, Cours au Collège de France. 1977-1978. Paris : Seuil.

Gil, J. (1985). Métamorphoses du corps, coll. Essais. Paris : Éditions de la Différence.

Gordimer, N. (1988). Where Do Whites Fit In? In The Essential Gesture : Writing, Politics and Places. Londres : J. Cape.

Lavigne, S. (2011). De la négritude à la migritude : une analyse sociologique de la littérature de l'Afrique francophone. Thèse. Université du Québec à Montréal, Doctorat en sociologie.

Lebris, M., \& Rouaud, J. (2007). Pour une littérature-monde. Paris : Gallimard.

Moura, J.-M. (1999). Littératures francophones et théories postcoloniales. Paris : PUF. 